\title{
Certainty Principle Using Complex Plane
}

\author{
Bhushan Poojary
}

\begin{abstract}
Heisenberg proved that uncertainty in measurement of position and momentum is because $(x . \dot{p}-\dot{p} \cdot x) \psi(x)=i \hbar \psi(x)$, i.e. position and momentum operator do not compute. Aim of the paper is to make position and momentum compute and prove uncertainty do not exists if the position and momentum is considered in complex plane. In this paper it is proved that if we take the position and momentum to complex plane, position and momentum operator compute, i.e. it is equal to zero. Similarly it is proved that if energy and time component are considered in complex plane uncertainty can be eliminated. This lead to conclusion that energy, time, position and velocity are not real values but complex values.
\end{abstract}

Index Terms-Heisenberg uncertainty principle, complex plane, certainty principle, Wirtinger derivatives.

\section{HeISENBERg UnCERTAINTY PRINCIPLE}

In Heisenberg uncertainty principle (in one dimension) value of psi $\psi$ is considers as

$$
\psi=e^{i(\mathrm{kx}-\omega \mathrm{t})}
$$

And momentum operator as

$$
\dot{p}=-i \hbar \frac{d}{d x}
$$

Heisenberg proved that

$$
(x \cdot \dot{p}-\dot{p} \cdot x) \psi(x)=i \hbar \psi(x)
$$

From above equations we can see that position and momentum do not compute, hence it was concluded that position $x$ and momentum $p$, cannot be known simultaneously. Werner Heisenberg stated that the more precisely the position of some particle is determined, the less precisely its momentum can be known, and vice versa [1].

So to remove uncertainty one would have to prove that position and momentum compute.

\section{COMPleX SPACE CoORdinates}

From paper Fine structure decoded [2], the space coordinates in 3 dimensions can be expressed as $(x, y, z)$. For simplicity, imagine an entity in one dimensional world just as shown in diagram below.

Manuscript received March 12, 2014; revised May 21, 2014.

Bhushan Poojary is with NIMS University, India (e-mail: bhushanpoojary@gmail.com).
Space coordinates for entity above can be written as $\left\{x_{r}\right\}$ and in vector notation $\vec{r}=x_{r} \vec{i}$

Now suppose space is curved in proximity of entity as shown below.

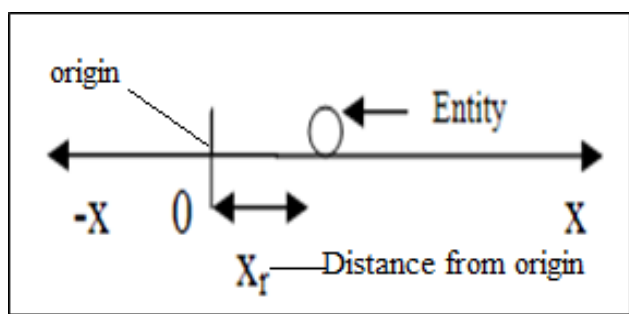

Fig. 1. Entity in one dimensional world.

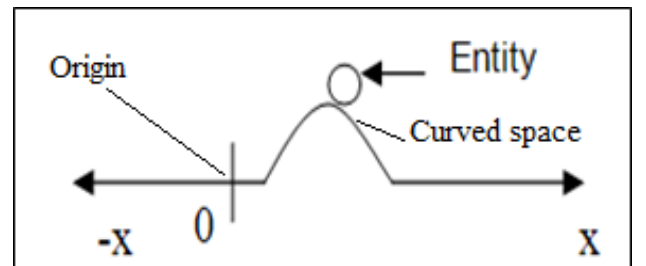

Fig. 2. Entity in 1 dimensional world but in curved space time.

If you want to find space coordinates you cannot use previous approach because space coordinate cannot be only expressed in terms of $\left\{x_{r}\right\}$ now you would think it's easy to express space coordinate in 2 dimension and express position in terms of $\{x, y\}$ but as told earlier entity is in one dimensional world. This can be resolved when we modify the diagram as shown below.

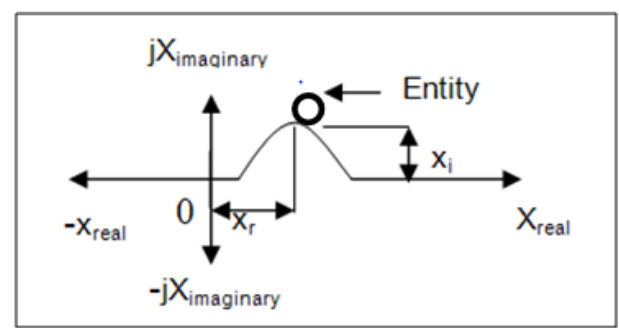

Fig. 3. Entity in 1 dimensional world but in curved space time.

Now we can say that entity is in one dimension but it's in complex plane. The space coordinates can now be expressed in real and imaginary number $\left\{x_{r}, x_{i}\right\}$.In terms of vector it can be written a $\vec{r}=x_{r} \vec{i}+j x_{i} \vec{i}$. Hence we can write space coordinates as $\{x r+j x i\}$ or $\{\langle x r, x i\rangle\}$

Now this can be extended easily to three dimensional world, space coordinate as

$$
\left\{x_{r}+j x_{i}, y_{r}+j y_{i}, z_{r}+j z_{i}\right\}
$$

And in Vector notation as 


$$
\vec{r}=\left(x_{r}+i x_{i}\right) \vec{i}+\left(y_{r}+i y_{i}\right) \vec{j}+\left(z_{r}+i z_{i}\right) \vec{k}
$$

From above Fig. 1-Fig. 3 we can say that electron/positron also moves in imaginary plane. So we should consider imaginary and real plane while computing position and momentum vectors to get overall picture.

\section{III. $\psi$ IN TERMS OF COMPLEX PLANE}

If we consider only one dimension we can express electron positron in terms of complex variable $\chi=x_{r}+i x_{i}$, where $x_{r}$ is position in real plane (external) and $x_{i}$ in imaginary plane (internal). So we can write $\Psi$ in terms of complex as shown below.

$$
\psi=e^{i(\mathrm{k} \chi-\omega \mathrm{t})}
$$

\section{Heisenberg EQuation in COMPlex Plane}

From Eq. (1) we have

$$
(x \cdot \dot{p}-\dot{p} \cdot x) \psi(x)=i \hbar \psi(x)
$$

where momentum operator is depended upon partial derivate of $\mathrm{x}$ and this $\mathrm{x}$ is what we measure i.e. the distance of element from the observer in real plane. From fine structure decoded paper it was proved that psi $\psi$ can be termed as electron movement in imaginary plane say $x_{i}$.

When electron is in imaginary plane by $\mathrm{x}_{\mathrm{i}}$ amount, than from the diagram below we can say that what we measure would byless than $x_{i}$. So we can say that $\bar{\gamma}=x_{r}-i x_{i}$. Where $\chi$ is the distance measured from the observer in complex plane (see Fig. 4).

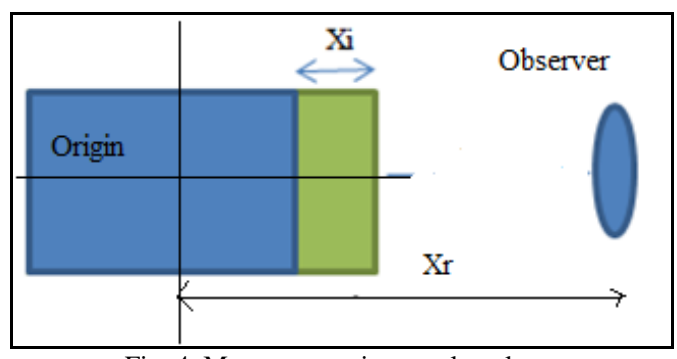

Fig. 4. Measurement in complex plane.

Actual measurement of the element from the observer would be $\chi=x_{r}-i x_{i}$. This is directlylinked to the element hence this should become the multiplying factor

Equation of Heisenberg in complex plane would look like this.

$$
\begin{gathered}
\left(\chi \frac{\partial}{\partial \bar{\chi}}-\frac{\partial}{\partial \bar{\chi}} \chi\right) \psi(x) \\
-\psi(x) \frac{\partial \chi}{\partial \bar{\chi}}
\end{gathered}
$$

\section{According to Wirtinger derivatives [3]}

$$
\frac{\partial}{\partial \bar{z}}=\frac{1}{2}\left(\frac{\partial}{\partial x}-i \frac{\partial}{\partial y}\right)
$$

Hence we can say that

$$
\frac{\partial f(\mathrm{z})}{\partial \bar{z}}=0
$$

which is to say that, roughly, $f$ is functionally independent from the complex conjugate of $z$.

From above equation we can say that

$$
-\psi(x) \frac{\partial \chi}{\partial \bar{\chi}}=0
$$

From Eq. (5) we can say that if we consider element in complex plane we can say there is no uncertainty in physics, as we have taken momentum operator and position to complex plane.

\section{SINGLE SLIT EXPLAINED}

When a monochromatic light is incident on a slit geometric optics predicts we will get a slit kind of image at the other side of the image as show in Fig. 5.

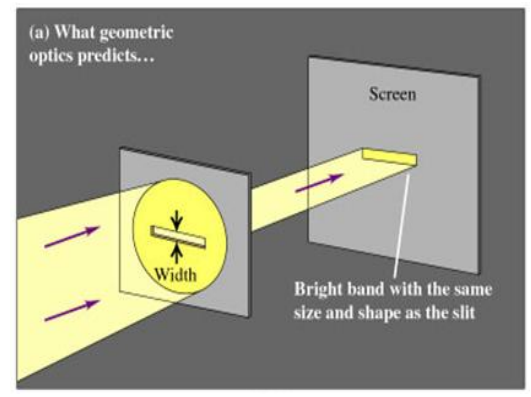

Fig. 5. Geometric optics prediction of single slit experiment.

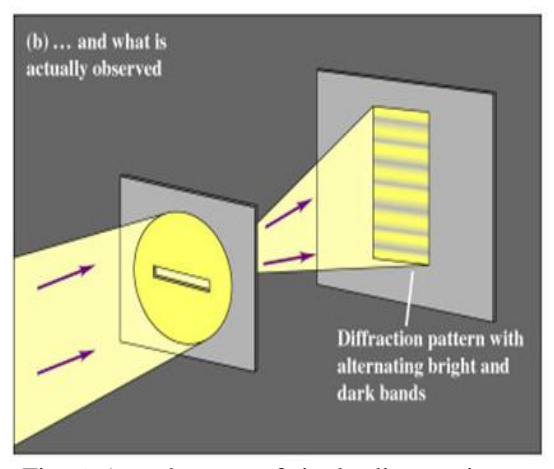

Fig. 6. Actual output of single slit experiment.

But actually it's not true actual picture is as shown below.

When the slit window is comparable to incident photon wavelength we get above output. This phenomenon was explained with help of Heisenberg uncertainty principle, by stating that photon exact momentum and position cannot be found precisely simultaneously. But with help of complex plane this phenomenon can be explained too. If you see Fig. 7, 
there is main spread of photon in centre. At $\mathrm{m} 1$ the measured photon momentum is $\mathrm{P}$ and at the centre its $p_{x}$. And vertical component is termed as $p_{y}$.

If you imagine photon travelling in $\mathrm{x}$ direction real plane and vibrating in complex plane say in $y$ axis.

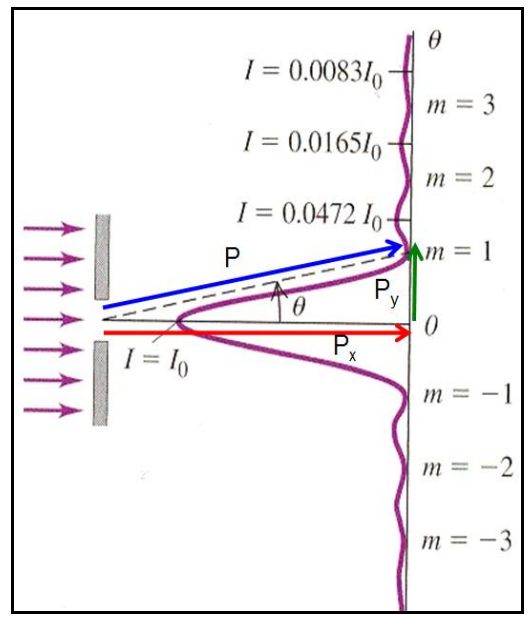

Fig. 7. Single slit intensity plot.

where momentum at $\mathrm{m} 1$ is written as

$$
\bar{p}=\bar{p}_{x}-\bar{p}_{y}
$$

But according to complex plane theory $p_{y}$ is $i p_{y i}$ That's why we can write

$$
\bar{p}=\bar{p}_{x}-i \bar{p}_{y i}
$$

Similarly if we consider all complex plane we get

$$
\bar{P}=\bar{p}_{X}+i p_{X}+\bar{p}_{Y}+i p_{Y I}+\bar{p}_{Z}+i p_{Z I}
$$

And position vector can be expressed as

$$
\bar{r}=\bar{x}_{R}+i x_{X}+\bar{y}_{R}+i y_{I}+\bar{z}_{R}+i z_{I}
$$

From above equations it is clear that momentum is not only in real plane but in complex plane. When we consider this we get diffraction pattern as predicted by Heisenberg.

\section{ENERGY TIME UNCERTAINTY EXPLANED}

Energy equation in terms of momentum is given be equation below [4]

$$
E^{2}=\left(m c^{2}\right)^{2}+(p c)^{2}
$$

In above equation only the real component of momentum is considered, if we consider imaginary plane as well above equation would look like this

$$
E^{2}=\left(m c^{2}\right)^{2}+(|P| c)^{2}
$$

where

$$
P=p_{r}+i p_{i} \text { and }|P|=\sqrt{p_{r}^{2}+p_{i}^{2}}
$$

Hence we can write energy equation as

$$
E^{2}=\left(m c^{2}\right)^{2}+\left(p_{r}{ }^{2}+p_{i}{ }^{2}\right)(c)^{2}
$$

From above equation it is clear that energy also has one real and one imaginary component.

Time is measured as inverse of frequency, frequency means vibration and vibrations in real world means the time component which we consider is only of real plane not of imaginary plane. Hence time can also be considered in complex plane as shown below.

$$
\tau=t_{r}+i t_{i}
$$

To prove energy time uncertainty wrong we will have to commute energy time in complex plane.

In real plane energy and time do not commute and equation looks like this.

$$
(t . \dot{E}-\dot{E} . t) \psi(x, t)=i \hbar \psi(x, t)
$$

where $\hat{E}$ energy operator termed as [5]

$$
\dot{E}=i \hbar \frac{\partial}{\partial t}
$$

If we consider we consider energy and time in complex plane equation would look like this.

$$
\begin{gathered}
=\left(\tau \frac{\partial}{\partial \bar{\tau}}-\frac{\partial}{\partial \bar{\tau}} \tau\right) \psi(x, t) \\
=-\psi(x, t) \frac{\partial \tau}{\partial \bar{\tau}}
\end{gathered}
$$

From Wirtinger derivatives equations as proved in position momentum the above term can be considered as zero.Hence we can say

$$
\left(\tau \frac{\partial}{\partial \bar{\tau}}-\frac{\partial}{\partial \bar{\tau}} \tau\right) \psi(x, t)=0
$$

From above we can conclude that energy time uncertainty vanishes if we consider energy and time in complex plane.

\section{QUANTUM TUNNELLING EFFECT EXPLAINED}

Quantum tunnelling effect is explained with help of Heisenberg uncertainty principle.

Our goal is to explain this effect with help of complex plane [6].

$\Psi(x)$ plot is shown below in tunnelling effect.

In above experiment energy $E$ of the particle is only the real component of the energy which is kinetic energy. As shown in Fig. 8 at the end of the barrier $\Psi(x)$ is $\Psi$ exit. So if 
we consider the $\Psi$ exit has the imaginary component $x_{i}$ at the exit, we can say there is momentum component in imaginary plane at the end of the barrier.

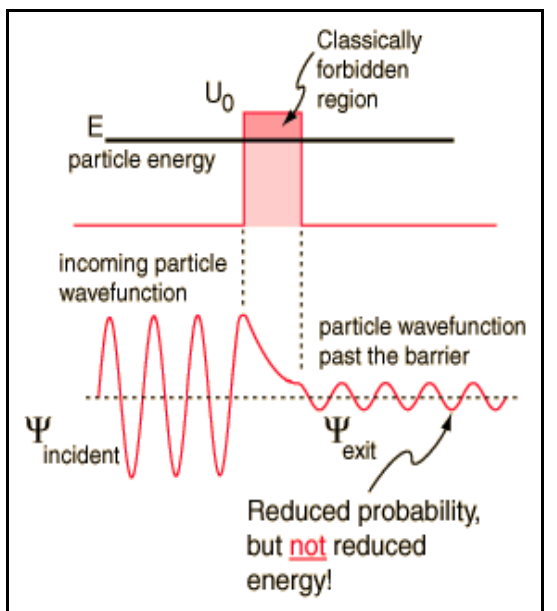

Fig. 8. Plot of $\Psi(x)$ in quantum tunnelling effect.

So we should consider this complex plane momentum because it adds additional energy to particle. Hence we can safely say that when complex energy of the particle is greater than the barrier potential the particle escape.

There is a probabilistic nature here because not all wave of particle will enter is same phase.
Similarly we can explain all phenomenon's in quantum mechanics which were explained with help of Heisenberg uncertainty principle with help of complex plane.

\section{REFERENCES}

[1] W. Heisenberg, "Über den anschaulichenInhalt der quanten theoret is chen Kinematik und Mechanik," Zeitschriftfür Physik, vol. 43, no. 3-4 pp. 172-198, 1927.

[2] B. Poojary, "Fine Structure Decoded," International Journal of Applied Physics and Mathematics, vol. 2, no. 4, pp. 244-249, 2012.

[3] A. Aldo, "Introduzioneall'analisicomplessa," Lezionitenutenelfebbraio, 1976.

[4] J. R. Forshaw and A. G. Smith, Dynamics and Relativity, Wiley, p. 149, 249, 2009.

[5] D. McMahon, Quantum Mechanics Demystified, USA: McGraw Hill, 2006, ISBN(10) 0071455469.

[6] N. Haibel, Zero Time Space, Wiley-VCH, p. 1, 2008.

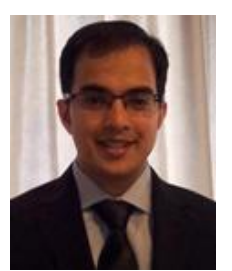

Bhushan Poojary is pursuing bachelor's degree from NIMS university. He was born in Mumbai on 26 July 1984. He has done bachelor of engineering in electronics and telecom from Mumbai University in year 2006. He is currently working as a software engineer in Credit Suisse in Mumbai. He has published paper fine structure decoded in IJAPM 2012, vol. 2, no. 4 